\title{
ORIGINAL
}

\section{CONCORDANCIA ENTRE LOS PORCENTAJES DE GRASA CORPORAL ESTIMADOS MEDIANTE EL ÁREA ADIPOSA DEL BRAZO, EL PLIEGUE DEL TRÍCEPS Y POR IMPEDANCIOMETRÍA BRAZO-BRAZO}

Vicente Martín Moreno (1), Juan Benito Gómez Gandoy (1), María Jesús Antoranz González (1) y Agustín Gómez de la Cámara (2)

(1) Centro de Salud Coronel de Palma, Móstoles.

(2) Unidad de Investigación y Epidemiología Clínica. Hospital Doce de Octubre, Madrid.

\section{RESUMEN}

Fundamento: La valoración del estado nutricional a partir de los miembros superiores es útil a nivel clínico. El objetivo de este trabajo es analizar el grado de acuerdo entre los porcentajes de grasa corporal (\% GC) estimados mediante el área adiposa del brazo BRAZO CEPS ) y por bioimpedancionetía brazo-brazo $\left(\% \mathrm{GC}_{\mathrm{BIA}}\right)$, así como con la ecuación de Siri para cuatro pliegues $\left(\% \mathrm{GC}_{\mathrm{SIRI}}\right)$.

Métodos: Estudio descriptivo transversal. Se valoró antropométricamente y por impedanciometría $\left(\mathrm{Omron} \mathrm{BF} 300^{\circ}\right)$ a 145 personas ( 83 varones, 62 mujeres). La concordancia entre métodos se analizó mediante el coeficiente de correlación intraclase (CCI) y el método de Bland-Altman

Resultados: el CCI entre \% $\mathrm{GC}_{\text {- }} \quad \% \mathrm{GC}_{\text {Ti }}$ fue de 0,8322 (intervalo de confianza al $95 \% 0,7672-0,8791$ ); entre $\% \mathrm{GCB}_{\mathrm{RA}}$ $\% \mathrm{GC}_{\mathrm{BA}}$ de $0,7337(0,6305-0,8080)$ y entre $\% \mathrm{GC}_{\mathrm{T}} \mathrm{CPPS}^{-} \% \mathrm{GC}_{\mathrm{BIZ}} \mathrm{de}$ $\% \mathrm{GC}_{\mathrm{BIA}}$ de $0,7337(0,6305-0,8080)$ y entre $\% \mathrm{GC}_{\mathrm{TRICEPS}} \% \mathrm{GC}_{\mathrm{BIA}}$ de
$0,9290(0,9015 ; 0,9488)$. Con el método de Bland-Altman el intervalo de concordancia entre $\% \mathrm{GC}_{\mathrm{BRAZO}} \% \mathrm{GC}_{\mathrm{TR}}{ }_{\mathrm{T} \text { CEPS }}(-11,2 ; 16,96)$ y entre $\% \mathrm{GC}_{\mathrm{BRAZO}}-\% \mathrm{GC}_{\mathrm{BI}}(-13,04 ; 21,76)$ superó el punto de corte $( \pm 10 \%)$, pero no entre $\% \mathrm{G}_{\mathrm{C}} \mathrm{Cog}^{-} \% \mathrm{GC}_{\mathrm{BI}}(-6,64 ; 9,6), \% \mathrm{GC}_{\mathrm{SPC}}$ $\% \mathrm{GC}_{\text {TRÍCEPS }}(-5,27 ; 4,52)$ y $\% \mathrm{GC}_{\text {SIRI }}{ }^{-} \% \mathrm{GC}_{\mathrm{BIA}}(-6,31 ; 8,52)$. El IMC no influyó en el resultado.

Conclusiones: El método utilizado influye en la valoración nutricional realizada a partir de los miembros superiores. El área adipara el pliegue del tríceps o para cuatro pliegues y a la impedanciometría, y aunque este resultado sugiere que el área adiposa del brazo puede no ser un indicador válido de la adiposidad global, este extremo deberá ser confirmado frente a una técnica patrón.

Palabras clave: Impedancia bioeléctrica. Antropometría. Composición corporal. Evaluación nutricional. Grosor de pliegues cutaneos.

Correspondencia:

Vicente Martín Moreno

Calle del Alerce 5, $5^{\circ} \mathrm{B}$

28041 Madrid

Teléfono 913698654

Correo electrónico amanvic@eresmas.com

\section{ABSTRACT}

Interchangeability among the Percentages of Body Fat Estimated by Mid-Arm Adipose Area, Triceps Skinfold Thickness and Arm-to-Arm Segmental Bioimpedance Analysis

Background: Assessing nutritional status based on the uppe limbs is useful at the clinical level. The aim of this study is to evaluate the agreement degree of the body fat percentages (\%BF) estimated by the mid-arm adipose area $\left(\% \mathrm{BF}_{\mathrm{AM}}\right)$, the Siri triceps skinfold equation ( $\left.\% \mathrm{BF}_{\mathrm{TR}} \mathrm{pS}\right)$ and the arm-to-arm segmental bioimpedance analysis $\left(\% \mathrm{BF}_{\mathrm{BL}}\right)$, as well as the Siri four-skinfold equation $\left(\% \mathrm{BF}_{\mathrm{SIRI}}\right)$, assessing their interchangeability.

Methods: A cross-sectional study. Body fat assessments were made on a total of 145 subjects ( 83 males, 62 females) anthropometrically and by bioelectrical impedance analysis (Omron BF $300^{\circ}$ ). The agreement between methods were analyzed using the interclass correlation coefficient (ICC) and the Bland-Altman method.

Results: The ICC between $\% \mathrm{BF}_{\mathrm{ARM}}$ and $\% \mathrm{BF}_{\mathrm{TR}}$ ic w was 0.8322 (CI 95\% 0.7672-0.8791); between \% $\mathrm{BF}$ TRICEPS 0.7337 (0.6305-0.8080) and between \% $\mathrm{BF}_{\text {TRICEPS }} \% \mathrm{BF}_{\mathrm{BIA}} 0.9290(0.9015$; 0.9488 ). For the Bland-Altman method, the agreement interval between $\% \mathrm{BF}_{\mathrm{ARM}^{-}}{ }^{-} \mathrm{BF}_{\mathrm{TRI}} \mathrm{CEPS}(-11.2 ; 16.96)$ and between $\% \mathrm{BF}_{\mathrm{ARM}}{ }^{-}$ $\% \mathrm{BF}_{\mathrm{BIA}}(-13.04 ; 21.76)$ exceeded the cutoff point $( \pm 10 \%)$, but not between $\% \mathrm{BF}_{\text {TRICEPS }}{ }^{-} \% \mathrm{BF}_{\text {BIA }}(-6.64 ; 9.6), \% \mathrm{BF}_{\text {SIRI }}{ }^{-} \% \mathrm{BF}_{\text {TRÍCEPS }}(-$ $5.27 ; 4.52)$ and $\% \mathrm{BF}_{\text {SIRI }^{-}}{ }^{-} \% \mathrm{BF}_{\mathrm{BIA}}(-6.31 ; 8.52)$. The $\mathrm{BMI}$ has no bearing on the results.

Conclusions: The method utilized influences the nutritional assessment made based on the upper limbs. The mid-arm adipose area method overestimated the \% BF with relationship to the Siri triceps skinfold or four-skinfold equation and to the bioelectrical impedance analysis, and although this result suggests that mid-arm adipose area may not be a good indicator of global adiposity, this must be confirmed against a gold standard.

Key words: Bioelectrical impedance. Anthropometry. Body composition. Body fat. Skinfold thickness. Nutrition Assessment. Arm. 


\section{INTRODUCCIÓN}

El estado nutricional influye en el mantenimiento de la salud y en la morbimortalidad asociada a múltiples procesos crónicos ${ }^{1-7}$, por lo que su valoración debe formar parte de la evaluación clínica del paciente. Esta valoración suele realizarse habitualmente desde una vertiente antropométrica, complementada con el análisis de diversos parámetros bioquímicos. El bajo coste y la accesibilidad de la antropometría justifican su predominio, relegando a un segundo plano a técnicas más precisas en la determinación de la composición corporal, como la densitometría y la tomografía axial computarizada $(\mathrm{TAC})^{8,9}$. Desde el punto de vista antropométrico el indicador más utilizado es el índice de masa corporal (IMC), quedando los pliegues cutáneos, la circunferencia del brazo, el perímetro muscular del brazo y otros indicadores nutricionales relegados, en general, a estudios epidemiológicos. Sin embargo, el IMC presenta limitaciones para determinar la composición corporal ${ }^{10-12}$, siendo conveniente en diversas situaciones clínicas cuantificar de forma más precisa la grasa corporal $^{1,6-8,13}$.

La estimación de la grasa corporal puede ser realizada por múltiples técnicas ${ }^{8,9}$ : densitometría, absorciometría de rayos $\mathrm{X}$ de dos energías (DEXA), resonancia magnética nuclear (RMN), TAC, etc..., pero sólo los pliegues cutáneos, la impedancia bioeléctrica y las ecuaciones basadas en medidas antropométricas son accesibles a todos los profesionales y pueden ser realizadas en la propia consulta.

El objetivo del presente estudio es analizar el grado de acuerdo entre tres métodos que utilizan la misma zona corporal, los brazos, para estimar el porcentaje de grasa corporal (\%GC): área adiposa del brazo, pliegue del tríceps mediante la ecuación de Siri e impedanciometría segmentaria brazo-brazo, valorar su intercambiabilidad y señalar los más convenientes en la evaluación nutricio- nal de una población adulta. De forma paralela, analizar la concordancia entre cada uno de estos métodos y el porcentaje de grasa corporal total estimado mediante la ecuación de Siri para cuatro pliegues.

\section{SUJETOS Y MÉTODOS}

Estudio descriptivo transversal realizado en los centros de salud Coronel de Palma y San Fernando de Móstoles. Ninguno de los tres métodos utilizados para estimar el porcentaje de grasa corporal a partir de los miembros superiores puede ser considerado como patrón oro, pero para la finalidad del estudio se utilizó como método de referencia el porcentaje de grasa corporal estimado a partir del área adiposa del brazo, al ser el más utilizado. Cada método se comparó además con un estimador de la adiposidad global, el porcentaje de grasa corporal calculado con la ecuación de Siri para cuatro pliegues cutáneos $(\mathrm{PC})^{14}$ :

$$
\% \mathrm{GC}_{\mathrm{SIRI}} \%=((4,95 / \mathrm{D})-4,5) \times 100
$$

siendo la densidad corporal (D):

$$
\begin{gathered}
\mathrm{D}=\mathrm{C}-\mathrm{M} \times \log _{10} \sum \text { 4PC (tríceps, bíceps, } \\
\text { subescapular y suprailíaco), }
\end{gathered}
$$

utilizando los coeficientes $\mathrm{C}$ y $\mathrm{M}$ de la suma de los cuatro pliegues específicos para cada grupo de edad en cada sexo reflejados de las tablas de Durnin y Womersley ${ }^{15}$.

Para calcular el tamaño de la muestra se tomó como referente la desviación estándar $(4,1 \%)$ del porcentaje de grasa corporal del estudio de Núñez y cols ${ }^{16}$, realizado sobre población de la misma comunidad autónoma, obteniendo, para una precisión de \pm una unidad en $\%$ GC y un intervalo de confianza del 95\%, un tamaño muestral de 72. Para disminuir el error tipo II fueron finalmente incluidas 145 personas sanas ( 83 varones, 62 mujeres). Todas las personas seleccionadas aceptaron participar. Los criterios utilizados 
para determinar las medidas antropométricas, ya fueron descritos en un estudio previo $^{17}$. Como parámetros antropométricos se determinaron peso, talla, IMC (peso $(\mathrm{kg}) /$ $\left.(\text { talla }(\mathrm{m}))^{2}\right)$, circunferencias de la cintura y del brazo y pliegues cutáneos del tríceps, bíceps, subescapular y suprailíaco. A partir del pliegue del tríceps (PT) y la circunferencia del brazo (CB), que se midió en el mismo punto que el pliegue, se calcularon el perímetro muscular del brazo (PMB):

$$
(\mathrm{PMB})=\mathrm{CB}-(3,14 * \mathrm{PT})
$$

el área muscular del brazo (AMB):

$$
(\mathrm{AMB})=(\mathrm{PMB})^{2} /(4 * 3,14)
$$

el área adiposa del brazo (AAB):

$$
\begin{gathered}
(\mathrm{AAB})=\mathrm{PT} \text { en } \mathrm{cm} *(\mathrm{CB} / 2)-((3,14 *(\mathrm{PT} \\
\text { en } \left.\left.\mathrm{cm})^{2}\right) / 4\right)
\end{gathered}
$$

y el porcentaje de grasa estimado a partir de dichas áreas $\left(\% \mathrm{GC}_{\mathrm{BRAZO}}\right)^{18}$ :

$$
\% \mathrm{GC}_{\text {brazo }}=(\mathrm{AAB} \times 100) /(\mathrm{AAB}+\mathrm{AMB})
$$

También se calculó el porcentaje de grasa corporal con la ecuación de Siri para el pliegue del tríceps $\left(\% \mathrm{GC}_{\mathrm{TRÍ}} \text { CEPS }\right)^{14}$ :

$$
\begin{gathered}
\% \mathrm{GC}_{\text {tríceps }}=\left(\left(4,95 / \mathrm{D}_{1}\right)-4,5\right) \times 100, \mathrm{con} \\
\mathrm{D}_{1}=\mathrm{C}-\mathrm{M} \times \log _{10} \mathrm{PT}
\end{gathered}
$$

Mediante el monitor OMRON BF $300^{\circledR}$ (OMRON Matsukasa Co. LTD, Japón) se obtuvo el porcentaje de grasa corporal por impedancia bioeléctrica $\left(\% \mathrm{GC}_{\mathrm{BIA}}\right)$. El monitor requiere para realizar la determinación el sexo, edad, estatura del sujeto y peso corporal. Las determinaciones se realizaron siguiendo las recomendaciones del fabricante y diversos estudios ${ }^{19,20}$, con al menos cuatro horas de ayuno y sin haber realizado ejercicio en las doce horas previas, por triplicado y anotando la media.

El análisis de los datos se realizó con el paquete estadístico SPSS para Windows ${ }^{\circledR}$ (versión 10.0.7; SPSS Inc. Chicago, Estados Unidos). Se obtuvo la media, desviación estándar y rango de todas las medidas, calculándose el coeficiente de correlación de Pearson entre los porcentajes de grasa corporal y el IMC y la circunferencia de la cintura. Se determinó la distribución normal de las diferencias de porcentaje de grasa con la prueba de Kolmogorov-Smirnov con la corrección de Lilliefors, contrastándose las medias de porcentaje de grasa mediante el análisis de la varianza de una vía o la prueba de la t de Student para datos apareados. El límite de significación estadística fue $\mathrm{p}<0,05$.

Para valorar la concordancia entre técnicas se utilizaron el coeficiente de correlación intraclase (CCI), modelo de efectos aleatorios, estableciendo como concordancia buena que el límite inferior del intervalo de confianza al 95\% sea mayor de 0,75, y el método de Bland y Altman ${ }^{21}$. Para establecer los criterios clínicos de valoración del grado de acuerdo con el método de Bland y Altman se tomó como referencia la variabilidad entre técnicas que clínicamente parece asumible, estableciendo como grado de acuerdo excelente que los límites del intervalo de concordancia no excedan de $\pm 5 \%$ y como grado de acuerdo bueno que dichos límites no excedan de $\pm 10 \%$.

\section{RESULTADOS}

Los datos antropométricos de los participantes se recogen en la tabla 1. Las diferencias en edad e IMC entre varones (38,6 \pm 12,6 años y $26,7 \pm 3,8$ respectivamente) y mujeres $(37,3 \pm 11,3$ años y $25,4 \pm 5,4)$ no fueron significativas. Las mujeres presentaron mayor cantidad de grasa que los varones, tanto a nivel local, como reflejan el área adiposa del brazo, el porcentaje de grasa obtenido a partir de dicha área $(39,5 \pm 7,98 \%$ frente a $22,7 \pm 8,3 \% ; \mathrm{p}<0,001)$, la ecuación de Siri para el pliegue del tríceps $(31,9 \pm 6,6 \%$ frente a $23,3 \pm 6,4 \%$; $\mathrm{p}<0,001)$ y el porcenta- 
Tabla 1

Datos antropométricos de los sujetos incluidos en el estudio. Media \pm desviación estándar. Entre paréntesis, intervalo. \% GC: porcentaje de grasa corporal.

\begin{tabular}{|c|c|c|c|}
\hline & Total & Varones & Mujeres \\
\hline Número de sujetos & 145 & 83 & 62 \\
\hline Edad (años) & $38,1 \pm 12,1(16-62)$ & $38,6 \pm 12,6(17-62)$ & $37,3 \pm 11,3(16-60)$ \\
\hline Peso $(\mathrm{kg})$ & $72,86 \pm 14,2(41-115,5)$ & $79,6 \pm 11,4(58,2-115,5)$ & $63,8 \pm 12,6(41-94,4)$ \\
\hline Talla $(\mathrm{cm})$ & $166,9 \pm 9,7(143,6-190)$ & $172,8 \pm 7,14(150-190)$ & $158,9 \pm 6,3(143,6-171,3)$ \\
\hline IMC & $26,1 \pm 4,6(15,5-38,6)$ & $26,7 \pm 3,8(19,1-38,5)$ & $25,4 \pm 5,4(15,5-38,6)$ \\
\hline Circunferencia de la cintura $(\mathrm{cm})$ & $89,1 \pm 13,9(59,6-127,0)$ & $95,1 \pm 10,9(73,6-127,0)$ & $81,2 \pm 13,6(59,6-124,0)$ \\
\hline Circunferencia del brazo & $29,3 \pm 4,1(19,7-40,3)$ & $30,5 \pm 2,9(23,4-40,3)$ & $27,6 \pm 4,8(19,7-37,2)$ \\
\hline Perímetro muscular del brazo $(\mathrm{cm})$ & $24,4 \pm 3,8(15,3-33,5)$ & $26,7 \pm 2,1(22,1-33,5)$ & $21,3 \pm 3,3(15,3-27,5)$ \\
\hline Área muscular del brazo $\left(\mathrm{cm}^{2}\right)$ & $48,6 \pm 14,2(18,6-89,4)$ & $57,1 \pm 9,3(39-89,4)$ & $37,2 \pm 11,3(18,6-60,1)$ \\
\hline Área adiposa del brazo $\left(\mathrm{cm}^{2}\right)$ & $21 \pm 11(2,5-55,2)$ & $17,6 \pm 9,1(2,5-55,2)$ & $25,5 \pm 11,8(6,5-51,4)$ \\
\hline $\begin{array}{l}\text { \%GC a partir de las áreas adiposa y } \\
\text { muscular del brazo }\left(\% \mathrm{GC}_{\text {brazo }}\right)\end{array}$ & $\begin{array}{l}29,9 \pm 11,6 \\
(4,8-57,6)\end{array}$ & $\begin{array}{l}22,7 \pm 8,3 \\
(4,8-49,1)\end{array}$ & $\begin{array}{c}39,5 \pm 7,98 \\
(20,98-57,6)\end{array}$ \\
\hline$\Sigma_{4}$ pliegues $(\mathrm{mm})$ & $59,4 \pm 25,2(11-124,5)$ & $54,2 \pm 22,2(11-122,5)$ & $66,2 \pm 27,5(19,8-124,5)$ \\
\hline $\begin{array}{l}\% \mathrm{GC} \text { con la ecuación de Siri para cuatro } \\
\text { pliegues }\left(\% \mathrm{GC}_{\text {Siri }}\right)\end{array}$ & $\begin{array}{c}26,6 \pm 8,3 \\
(4,5-44,7)\end{array}$ & $\begin{array}{l}22,7 \pm 6,5 \\
(4,5-37,3)\end{array}$ & $\begin{array}{c}31,8 \pm 7,6 \\
(13,9-44,7)\end{array}$ \\
\hline Pliegue del triceps $(\mathrm{mm})$ & $15,5 \pm 7,2(2,0-32,3)$ & $12,1 \pm 5,6(2,0-31,2)$ & $20,1 \pm 6,7(7,0-32,3)$ \\
\hline $\begin{array}{l}\text { \%GC con Siri para el pliegue del tríceps } \\
\left(\% \mathrm{GC}_{\text {tríceps }}\right)\end{array}$ & $\begin{array}{c}26,99 \pm 7,77 \\
(5,9-44,3) \\
\end{array}$ & $\begin{array}{c}23,3 \pm 6,4 \\
(5,9-37,04) \\
\end{array}$ & $\begin{array}{r}31,97 \pm 6,6 \\
(14,2-44,3) \\
\end{array}$ \\
\hline Grasa corporal Omron (\%GC & $25,5 \pm 8,9(4,1-44,9)$ & $21,7 \pm 7,1(4,1-38,4)$ & $30,6 \pm 8,5(6,6-44,9)$ \\
\hline
\end{tabular}

je de grasa obtenido por impedancia bioeléctrica $(30,6 \pm 8,5 \%$ frente a $21,7 \pm 7,1 \%$; $\mathrm{p}<0,001)$; como a nivel global, como muestra el porcentaje de grasa estimado mediante la ecuación de Siri para cuatro pliegues $(31,8$ $\pm 7,6 \%$ frente a $22,7 \pm 6,5 \% ; \mathrm{p}<0,001)$.

El porcentaje de grasa estimado mediante el área adiposa del brazo $(29,9 \pm 11,6 \%)$ fue significativamente más elevado $(\mathrm{p}<0,001)$ que el obtenido con la ecuación de Siri para el pliegue del tríceps $(26,99 \pm 7,77 \%)$ o por impedancia bioeléctrica $(25,5 \pm 8,9 \%)$ y también que el porcentaje de grasa corporal total estimado mediante la ecuación de Siri para cuatro pliegues $(26,6 \pm 8,3 \%)$. También alcanzaron significación estadística $(\mathrm{p}<0,001)$ las diferencias entre $\% \mathrm{GC}_{\mathrm{TRI}} \mathrm{CEPS}$ y $\% \mathrm{GC}_{\mathrm{BIA}}$. Sin embargo, $\% \mathrm{GC}_{\mathrm{TRÍC}}$ no difirió de forma significativa de $\% \mathrm{GC}_{\mathrm{SIRI}}$. $\mathrm{El}$ área adiposa del brazo aportó el porcentaje de grasa más elevado, de forma más acusada en mujeres, mientras que la menor media se obtuvo en uno y otro sexo mediante impedancia bioeléctrica con el monitor Omron BF $300^{\circledR}$ (tabla 1). 
Respecto al IMC, el porcentaje de grasa que se asoció de forma más intensa con dicho parámetro fue el $\% \mathrm{GC}_{\mathrm{BIA}}$ en las mujeres y el \% $\mathrm{GC}_{\mathrm{SIRI}}$ en los varones (tabla 2), mientras que la asociación menos intensa la estableció el \% $\mathrm{GC}_{\mathrm{BRAzO}}$ en ambos sexos. Con la circunferencia de la cintura el porcentaje de grasa que mejor correlacionó en ambos sexos fue el $\% \mathrm{GC}_{\mathrm{BIA}}$, mientras que entre $\% \mathrm{GC}_{\mathrm{BRAZO}}$ y circunferencia de la cintura no se alcanzó significación estadística cuando se analizaron de forma global los datos, resultado que también se observó cuando se comparó dicho porcentaje de grasa y la circunferencia del brazo.

Al analizar el grado de acuerdo se observó (tablas 3 y 4) que el coeficiente de correla- ción intraclase $(\mathrm{CCI})$ entre $\% \mathrm{GC}_{\mathrm{BRAZO}} \mathrm{y}$ $\% \mathrm{GC}_{\mathrm{TR} i ́ c E P S}$ fue de 0,8322 ; entre $\% \mathrm{GC}_{\mathrm{BRAZO}}$ y $\% \mathrm{GC}_{\mathrm{BIA}}$ de 0,7337 , con límite inferior del intervalo de confianza al $95 \%$ por debajo de $0,75(0,6305)$; y entre $\% \mathrm{GC}_{\text {TRICEPS }} \mathrm{y} \% \mathrm{G}_{\text {CBIA }}$ de 0,9290 . Entre $\% \mathrm{GC}_{\text {BRAZO }} \mathrm{y} \% \mathrm{GC}_{\mathrm{SIRI}}$ el límite inferior del intervalo de confianza también se sitúa por debajo de 0,75 , por lo que el grado de acuerdo tampoco alcanza la categoría de bueno. Al analizar los resultados por sexo, sólo $\% \mathrm{GC}_{\text {TRICEPS }}-\% \mathrm{GC}_{\mathrm{BIA}}$; $\% \mathrm{G}_{\text {CBIA }}-\% \mathrm{GC}_{\text {SIRI }}$ y $\% \mathrm{GC}_{\text {TRICEPS }}-\% \mathrm{GC}_{\text {SIRI }}$ mantienen dichos límites inferiores por encima de 0,75 .

Mediante el método de Bland y Altman, los límites del intervalo de concordancia entre $\% \mathrm{GC}_{\text {BRAZO }}$ y $\% \mathrm{G}_{\text {CTRÍCEPS }}$ (tabla 3 , in-

Tabla 2

Coeficientes de correlación de Pearson entre los porcentajes de grasa corporal estimados mediante pliegue de triceps, área adiposa del brazo e impedancia bioeléctrica y el porcentaje de grasa corporal estimado mediante cuatro pliegues ( $\% \mathrm{GC}_{\mathrm{Siri}}$ ), el IMC y las circunferencias de la cintura y del brazo.

\begin{tabular}{|c|c|c|c|c|}
\hline \multicolumn{5}{|c|}{ Coeficientes de correlación de Pearson } \\
\hline Global & $\% G_{\text {brazo }}$ & $\% \mathrm{GC}_{\text {Siri }}$ & $\% \mathbf{G C}_{\text {tríceps }}$ & $\% \mathrm{GC}_{\text {BIA }}$ \\
\hline$\% \mathrm{GC}_{\text {Siri }}$ & $0,765^{* *}$ & - & $0,956^{* *}$ & $0,909^{* *}$ \\
\hline IMC & $0,257^{* *}$ & $0,613^{* *}$ & $0,547^{* *}$ & $0,660^{* *}$ \\
\hline Circunferencia de la cintura & $-0,071$ & $0,344^{* *}$ & $0,288^{* *}$ & $0,394^{* *}$ \\
\hline Circunferencia del brazo & 0,077 & $0,394^{* *}$ & $0,344^{* *}$ & $0,367^{* *}$ \\
\hline Varones & $\% \mathbf{G C}_{\text {brazo }}$ & $\% \mathrm{GC}_{\text {Siri }}$ & $\% \mathbf{G C}_{\text {triceps }}$ & $\% \mathbf{G C}_{\text {BIA }}$ \\
\hline$\% \mathrm{GC}_{\text {Siri }}$ & $0,640^{* *}$ & - & $0,940^{* *}$ & $0,857^{* *}$ \\
\hline IMC & $0,569^{* *}$ & $0,802^{* *}$ & $0,725^{* *}$ & $0,783^{* *}$ \\
\hline Circunferencia de la cintura & $0,568^{* *}$ & $0,865^{* *}$ & $0,809^{* *}$ & $0,865^{* *}$ \\
\hline Circunferencia del brazo & $0,576^{* *}$ & $0,635^{* *}$ & $0,564^{* *}$ & $0,481^{* *}$ \\
\hline Mujeres & $\% G_{\text {brazo }}$ & $\% \mathrm{GC}_{\text {Siri }}$ & $\% \mathrm{GC}_{\text {triceps }}$ & $\% \mathrm{GC}_{\text {BIA }}$ \\
\hline$\% \mathrm{GC}_{\text {Siri }}$ & $0,649^{* *}$ & - & $0,937^{* *}$ & $0,897^{* *}$ \\
\hline IMC & $0,482^{* *}$ & $0,857^{* *}$ & $0,808^{* *}$ & $0,908^{* *}$ \\
\hline Circunferencia de la cintura & $0,373^{* *}$ & $0,828^{* *}$ & $0,763^{* *}$ & $0,846^{* *}$ \\
\hline Circunferencia del brazo & $0,461^{* *}$ & $0,834^{* *}$ & $0,822^{* *}$ & $0,802^{* *}$ \\
\hline
\end{tabular}

$* *: \mathrm{p}<0,01$. 


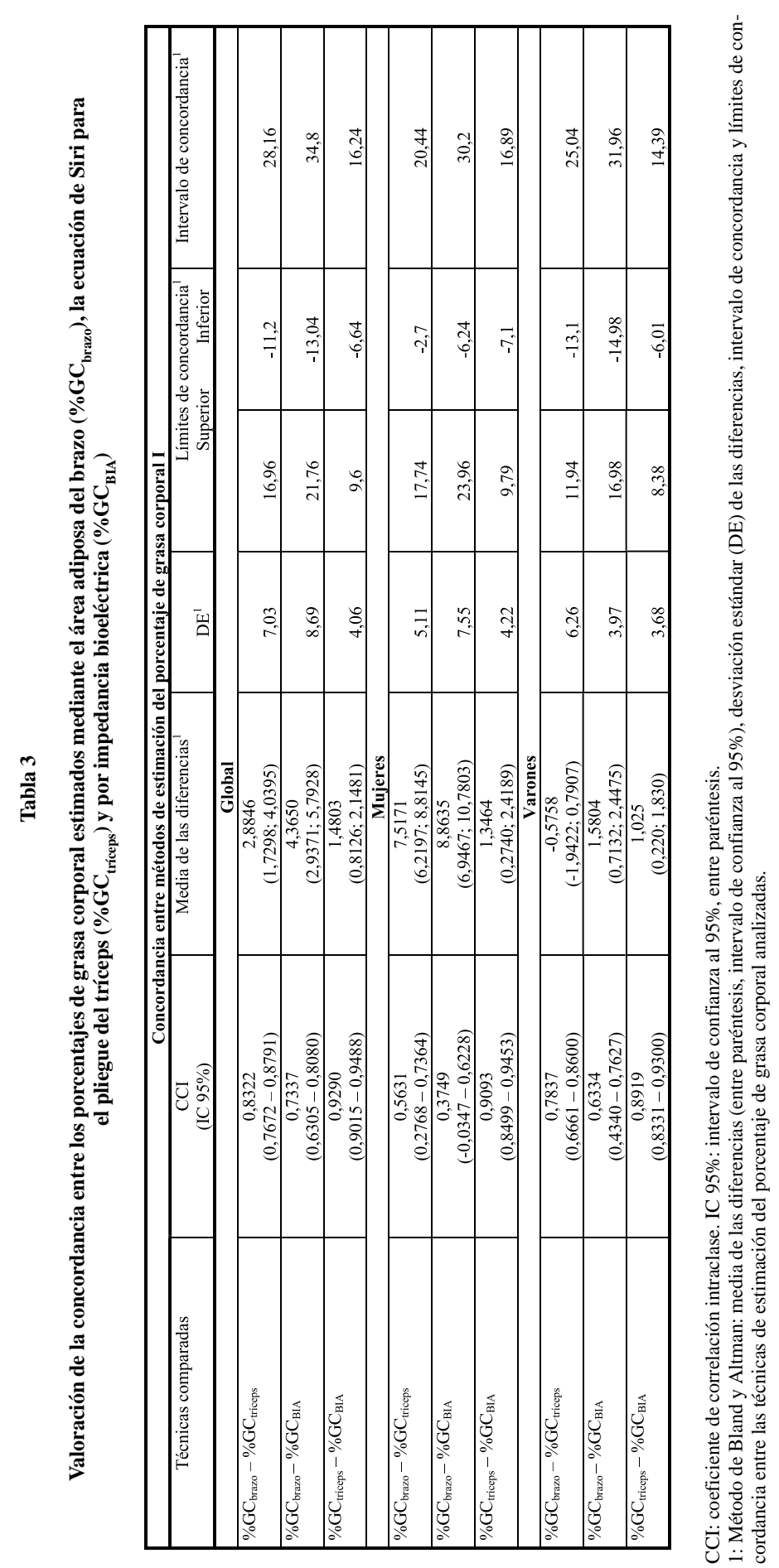


CONCORDANCIA ENTRE LOS PORCENTAJES DE GRASA CORPORAL ESTIMADOS MEDIANTE EL ÁREA...

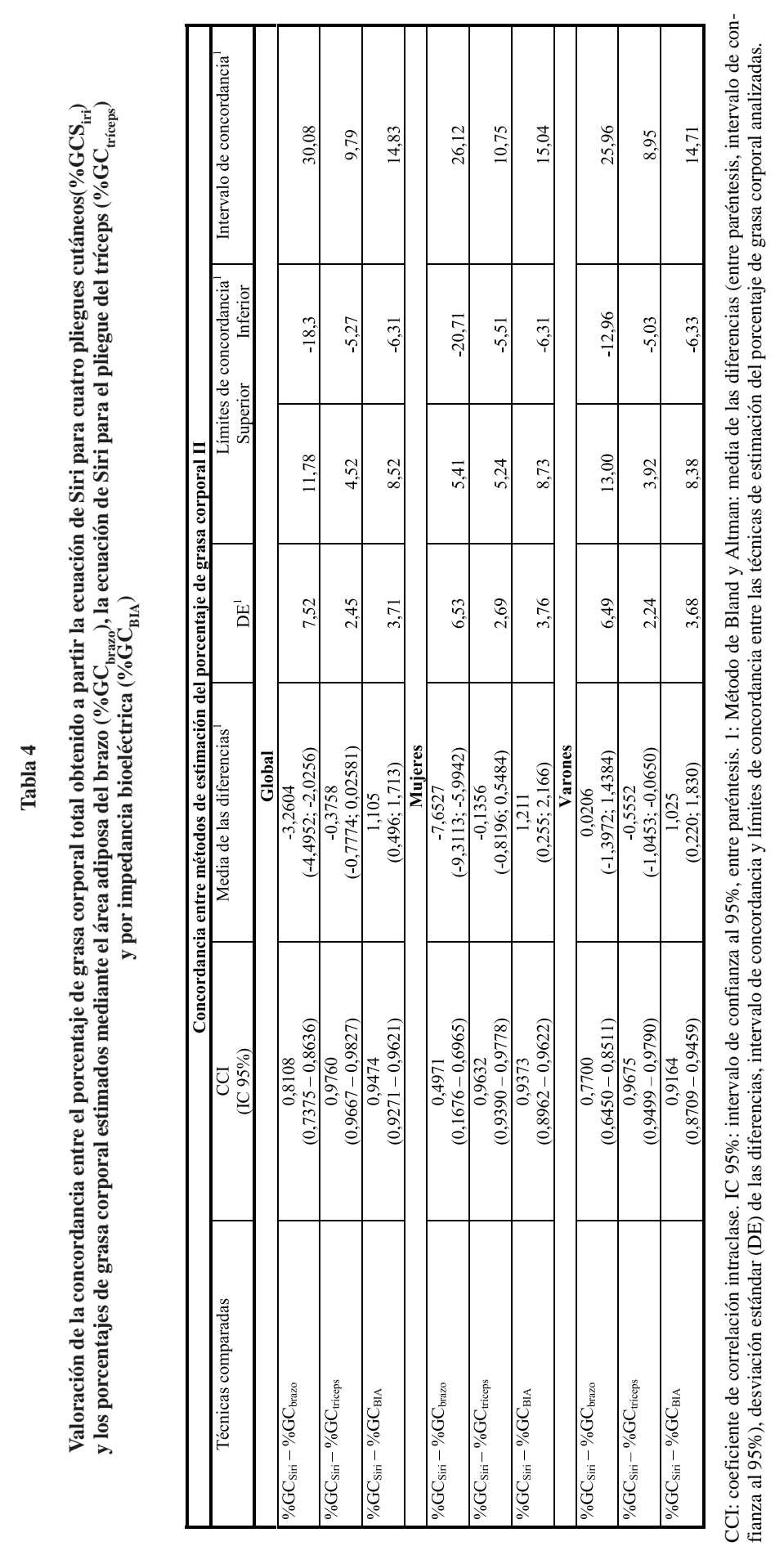

Rev Esp Salud Pública 2003, Vol. 77, N. ${ }^{\circ} 3$ 
Figura 1

Valoración mediante el método de Bland y Altman de la concordancia entre los porcentajes de grasa corporal estimados mediante la ecuación de Siri para el pliegue del tríceps $\left(\% \mathrm{GC}_{\mathrm{Sir} \text {-tríceps }}\right)$, el área adiposa de brazo $\left(\% \mathrm{GC}_{\text {brazo }}\right)$ y por impedancia bioeléctrica $\left(\% \mathrm{GC}_{\text {Omron }}\right)$ en varones y mujeres con IMC menor de 30
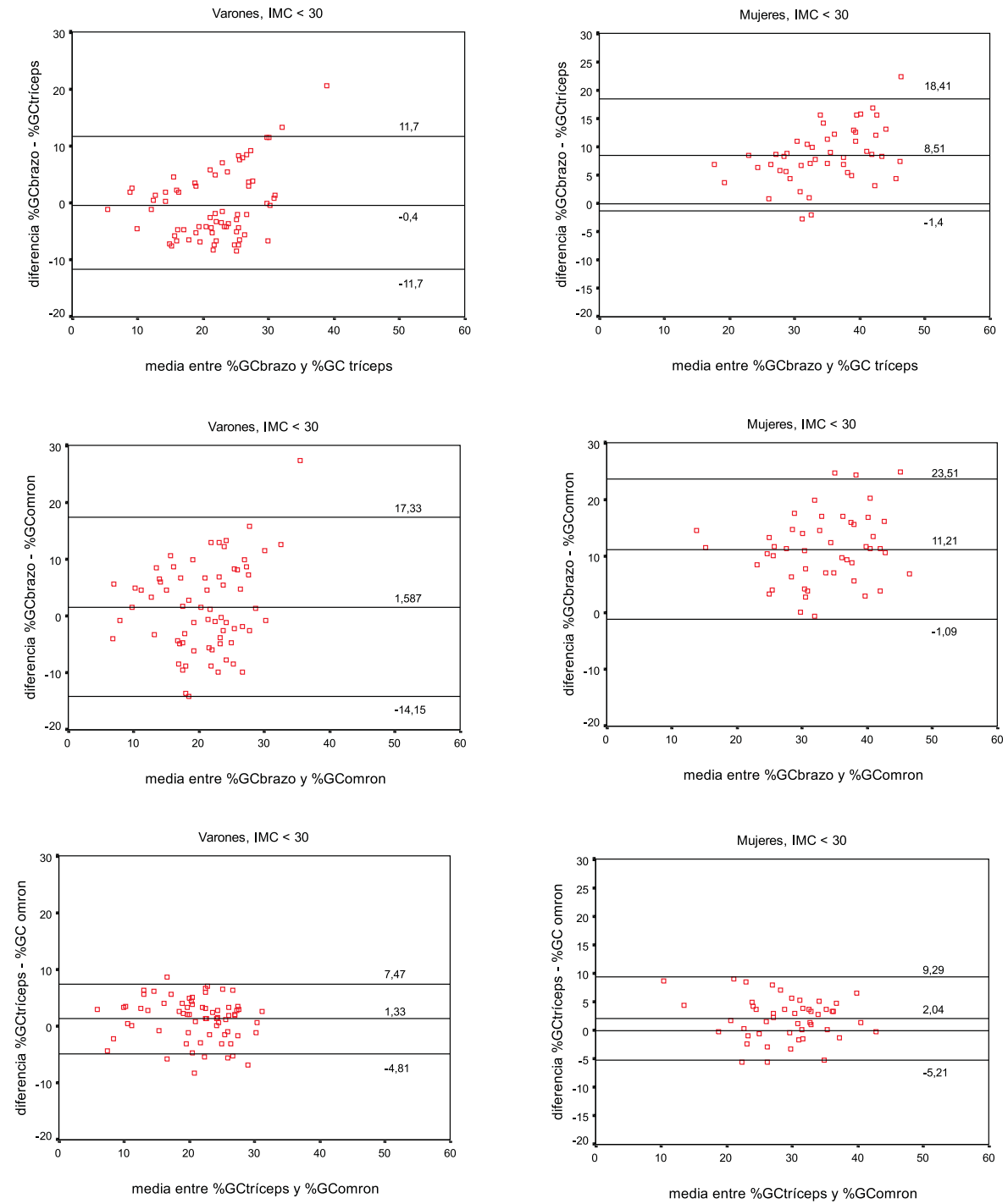

tervalo entre $-11,2$ y 16,96$)$, entre $\% \mathrm{GC}_{\mathrm{BRA}}$ zO $\mathrm{y} \% \mathrm{G}_{\mathrm{CBIA}}(-13,04 ; 21,76)$ y entre $\% \mathrm{GC}_{\mathrm{SI}-}$ RI $\mathrm{y} \% \mathrm{GC}_{\text {BRAZO }}$ (tabla 4 , intervalo entre $-18,3$ y 11,78$)$ superaron el punto de corte establecido para considerar el acuerdo como bueno $( \pm 10 \%)$. Sin embargo, entre $\% \mathrm{GC}_{\mathrm{TRICEPS}} \mathrm{y}$

$\% \mathrm{G}_{\mathrm{CBIA}}$ (media de las diferencias 1,48 \pm $4,06 \%$, intervalo de concordancia entre 6,64 y $9,6 \%$ ), entre $\% \mathrm{GC}_{\text {SIRI }}$ y $\% \mathrm{GC}_{\mathrm{TRICEPS}}$ (media de las diferencias $-0,3758 \pm 2,45 \%$, intervalo de concordancia entre $-5,268$ y $4,516 \%$ ) y entre $\% \mathrm{GC}_{\mathrm{SIRI}}$ y el porcentaje de 
Figura 2

Valoración mediante el método de Bland y Altman de la concordancia entre los porcentajes de grasa corporal estimados mediante la ecuación de Siri para el pliegue del tríceps ( $\% \mathrm{GC}_{\text {tríceps }}$ ), el área adiposa del brazo $\left(\% \mathrm{GC}_{\text {brazo }}\right)$ y por impedancia bioeléctrica $\left(\% \mathrm{GC}_{\mathrm{Omron}}\right)$ en varones y mujeres con IMC mayor o igual a 30
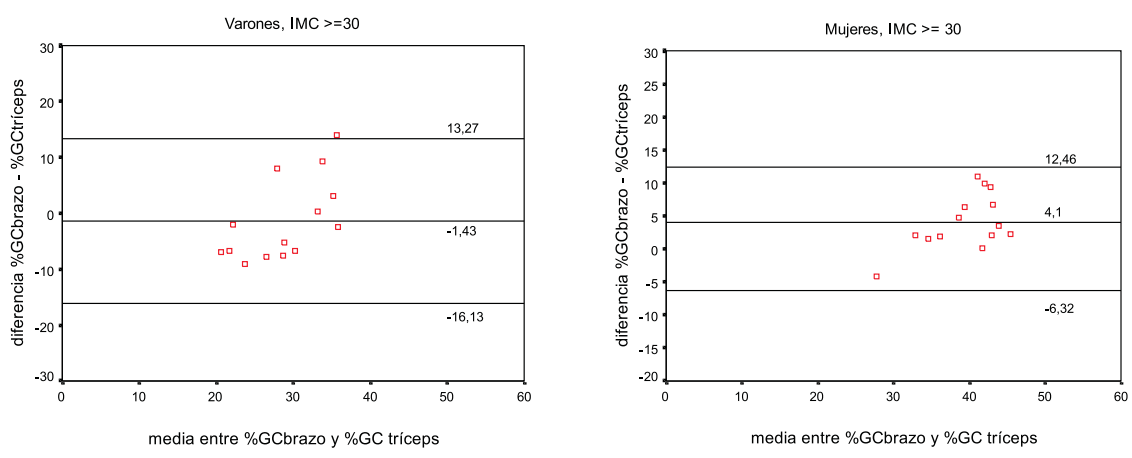

Varones, IMC $>=30$
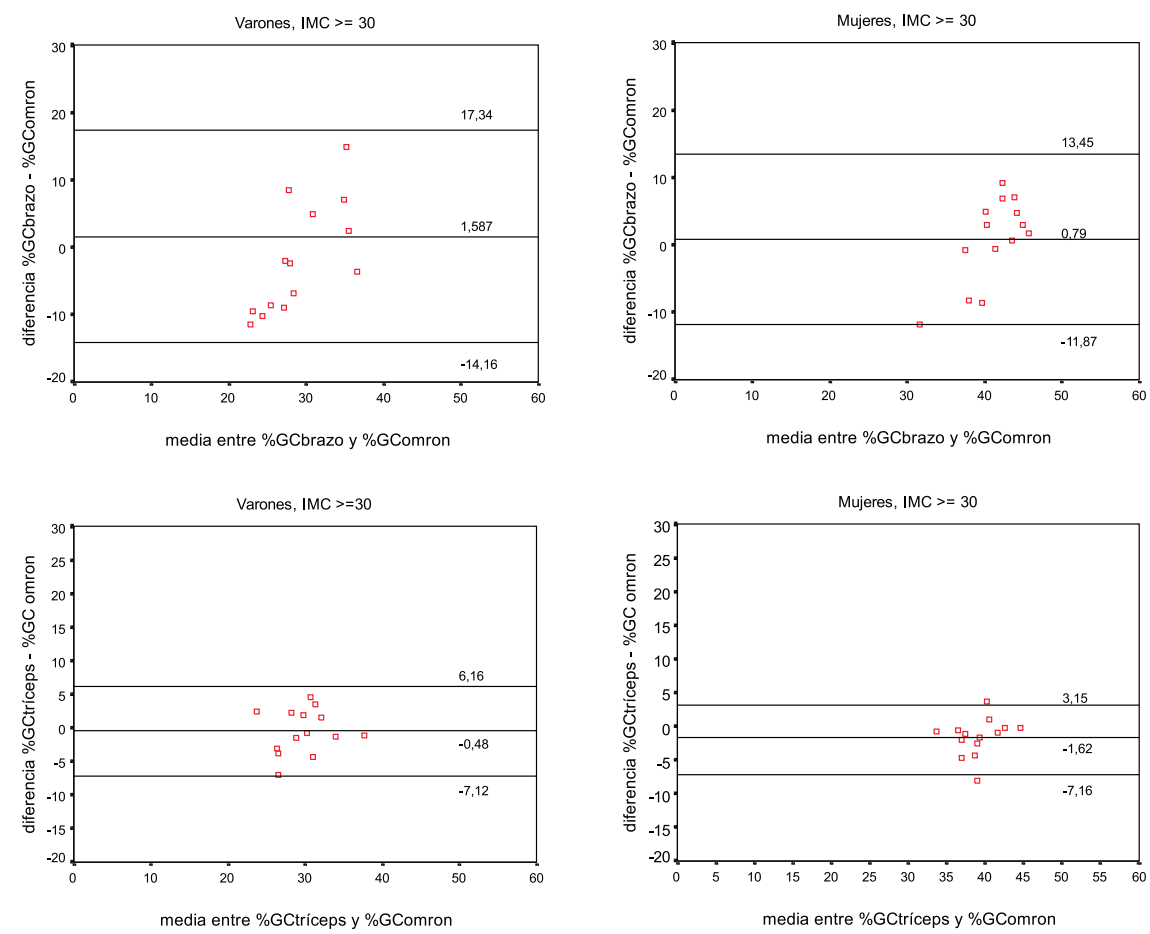

grasa obtenido por impedancia bioeléctrica con el monitor Omron BF $300^{\circledR}$ (media de las diferencias $1,105 \pm 3,71 \%$, intervalo de concordancia entre $-6,31$ y $8,52 \%$ ) se alcanzó un grado de acuerdo bueno (tabla 4), aunque entre $\% \mathrm{GC}_{\text {SIRI }}$ y $\mathrm{GC}_{\text {BIA }}$ la media de las

diferencias difiere significativamente de cero, indicando que el $\% \mathrm{GC}_{\mathrm{BIA}}$ tiende a subestimar el porcentaje de grasa corporal respecto al \% $\mathrm{GC}_{\mathrm{SIRI}}$.

Estos resultados no se modificaron cuando se dividió la población estudiada en obe- 
sos $($ IMC $\geq 30)$ y no obesos $($ IMC < 30) (gráficos 1 y 2). La diferencia entre $\% \mathrm{GC}_{\mathrm{BRAZO}}$ y el resto de porcentajes de grasa corporal es más acusada en las mujeres (tabla 1), sobre todo en las no obesas (gráfi$\cos 1$ y 2), observándose que aumenta a medida que aumenta el $\% \mathrm{GC}_{\mathrm{BRAZO}} \mathrm{y}$ que se atenúa con la edad (gráfico 3). Con relación a los parámetros que intervienen en el cálculo del área adiposa del brazo, se observa que la diferencia no aumenta al aumentar la circunferencia del brazo, pero sí lo hace al aumentar el pliegue del tríceps, resultado que es independiente del sexo. Sin embargo, cuando comparamos $\% \mathrm{GC}_{\mathrm{TR} \text { ĆEPS }}$ con $\% \mathrm{GC}_{\mathrm{BIA}}$ o con $\% \mathrm{GC}_{\mathrm{SIRI}}$ las diferencias no aumentan al aumentar el pliegue del tríceps (gráfico 4).

\section{DISCUSIÓN}

Aunque con los métodos utilizados el cálculo del porcentaje de grasa corporal se realiza de forma indirecta ${ }^{8,9}$, esta limitación incide de forma mínima en los objetivos del estudio, en primer lugar porque todos los métodos se basan en un modelo bicompartimental; en segundo lugar, porque, a excepción de la ecuación de Siri para cuatro pliegues, todos evalúan la grasa corporal a partir de estimaciones realizadas en los miembros superiores; en tercer lugar, porque parte de ellos comparten parámetros y esto permite evaluar qué componentes marcan las posibles diferencias; $y$, en cuarto lugar, porque con algunos métodos las medidas se han realizado en los mismos puntos corporales, con

Figura 3

Influencia de las variables porcentaje de grasa corporal a partir de las áreas del brazo, edad, circunferencia del brazo y pliegue del tríceps en la diferencia observada entre los porcentajes de grasa corporal estimados a partir del área adiposa del brazo y la ecuación de Siri para el pliegue del tríceps
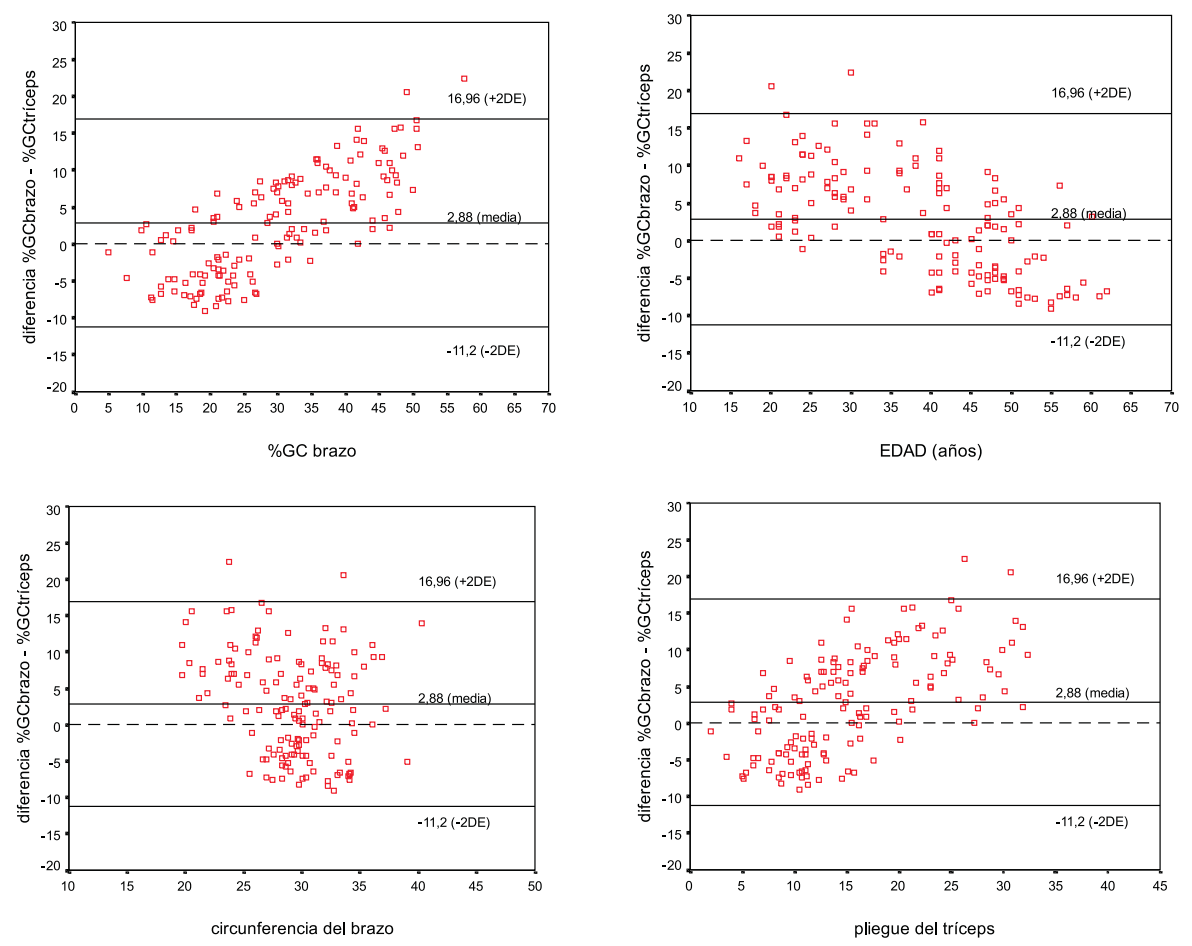
Figura 4

Influencia del pliegue del tríceps en las diferencias entre \% $\mathrm{GC}_{\mathrm{SIRI}}-\% \mathrm{GC}_{\mathrm{BIA}}, \% \mathrm{GC}_{\mathrm{SIRI}}-\% \mathrm{GC}_{\mathrm{TRI}}$ У \% GC TRÍCEPS $^{-\% \mathrm{GC}_{\text {BIA }}}$
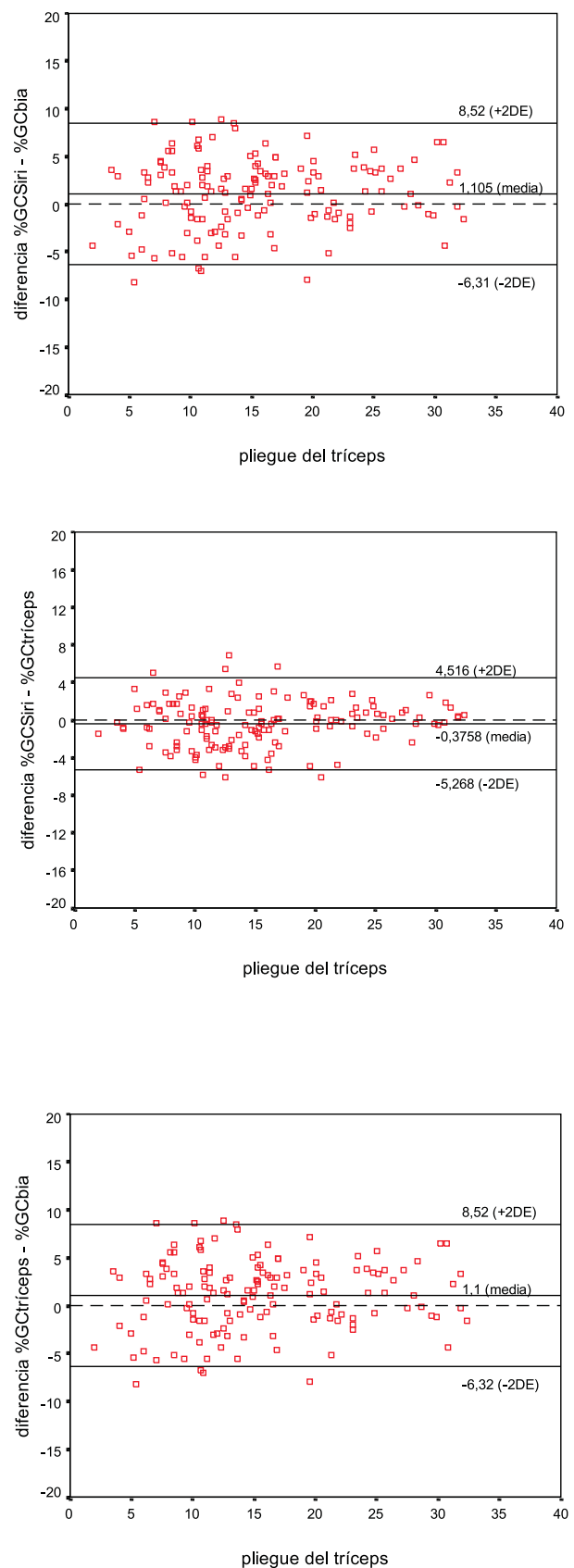

Rev Esp Salud Pública 2003, Vol. 77, N. ${ }^{\circ} 3$ lo que las posibles diferencias no se deben a estimaciones realizadas en zonas diferentes sino a la propia técnica.

Para el cálculo del porcentaje de grasa corporal a partir de las áreas muscular y adiposa del brazo se tomó como referente la recomendación de Alastrué y cols. ${ }^{18}$, refrendada por otros autores ${ }^{22}$, que sugiere que el área muscular del brazo puede ser utilizada para la estimación de la masa libre de grasa, y un modelo bicompartimental.

Con relación a la impedanciometría, esta técnica, basándose en las diferentes características conductoras de la masa grasa y de la masa libre de grasa debido a su diferente grado de hidratación ${ }^{19,20}$, realiza el cálculo del agua corporal total $\mathrm{y}$, a partir de este parámetro, estima la masa libre de grasa, que restada del peso corporal permite el cálculo del peso y porcentaje de grasa corporal. Depende con ello de diversas estimaciones, con sus consiguientes fuentes de error. Sin embargo, su aplicación en segmentos corporales ha demostrado una buena concordancia frente a impedanciometría corporal global y otras técnicas de referencia ${ }^{23,24}$ y, en concreto, el monitor Omron BF 300 ha sido validado frente a densitometría ${ }^{25}$ y pliegues cutáneos $^{17,20}$.

Por último, sobre la posible influencia en los resultados del lado corporal en que se realizan las medidas antropométricas, diversos estudios no han observado diferencias significativas entre los valores obtenidos en uno u otro lado $^{26,27}$.

Teniendo en cuenta las consideraciones previas, el análisis de la concordancia entre métodos permite observar que a nivel de grupo los porcentajes de grasa estimados mediante la ecuación de Siri para el pliegue del tríceps, por impedanciometría y la ecuación de Siri para cuatro pliegues, son intercambiables.

Sin embargo, el grado de acuerdo alcanzado entre el porcentaje de grasa estimado a 
partir de las áreas del brazo y los restantes métodos evaluados no permite su intercambiabilidad. Diversos factores, como las propias ecuaciones utilizadas, diferencias constitucionales entre ambos sexos, cambios relacionados con la edad e incluso factores de tipo étnico, pueden contribuir, de forma aislada o en combinación, a estas diferencias $^{18,26,28-32}$.

En este estudio $\% \mathrm{GC}_{\text {BRAZO }} \mathrm{y} \% \mathrm{GC}_{\text {TRÍcEPS }}$, que evalúan la misma zona corporal del brazo, presentan importantes diferencias, observándose que en los varones existe una importante variabilidad en el resultado ofrecido, reflejada en la amplitud del intervalo de concordancia, asociada en las mujeres a una sobreestimación del porcentaje de grasa corporal, y mientras que el $\% \mathrm{GC}_{\mathrm{TR}}$ ÍCEPS intercambiable con el $\% \mathrm{GC}_{\text {SIRI }}$, el $\% \mathrm{GC}_{\text {BRA- }}$ zo no lo es, resultado que sugiere que su utilización como estimador de la adiposidad global puede no ser válida. Diversos autores han descrito la influencia de la utilización de uno o más parámetros antropométricos y de la ecuación utilizada en el porcentaje de grasa corporal estimado ${ }^{17,32}$. Así, para calcular el \% $\mathrm{GC}_{\mathrm{TRÍCEPS}}$ sólo es necesario medir el pliegue del tríceps, mientras que para calcular el \% $\mathrm{GC}_{\mathrm{BRAZO}}$ es necesario medir además la circunferencia del brazo. La presencia de este segundo parámetro en las ecuaciones de las áreas muscular y adiposa utilizadas para calcular el \% $\mathrm{GC}_{\text {BRAZO }}$ y su posición en dichas ecuaciones posibilita por sí misma una variabilidad en el resultado ofrecido, ya que si mantenemos constante el valor del pliegue del tríceps el aumento de la circunferencia del brazo condiciona mayor incremento del área muscular que del área adiposa, con lo que el \% $\mathrm{GC}_{\mathrm{BRAZO}}$ estimado es menor. Por otro lado, se observa también que al aumentar el pliegue del tríceps aumenta la diferencia entre $\% \mathrm{GC}_{\text {BRAZO }}$ y $\% \mathrm{GC}_{\text {TRÍCEPS }}$, por lo que el modo en que la ecuación gestiona el pliegue del tríceps también influye en el porcentaje de grasa corporal obtenido.

En definitiva, ambos factores, número de parámetros antropométricos incluidos y ecuación utilizada, favorecen que el cálculo del porcentaje de grasa corporal ofrezca un valor más elevado cuando se realiza a partir de las áreas del brazo, pero no explican por sí solos las diferencias entre varones y mujeres.

Como en otros estudios, los varones presentan una mayor circunferencia del brazo que las mujeres, mientras que estas tienen un mayor pliegue del tríceps y mayor área adiposa del brazo ${ }^{18,26,31,34}$, de forma que el factor constitucional influye en que la diferencia respecto al método de referencia sea menor en los varones que en las mujeres, pero no explica los cambios observados con la edad.

En este sentido, y aunque no se dispone de estudios longitudinales en población de nuestro entorno, estudios transversales ${ }^{18,31,34}$ realizados con una separación de una década muestran un patrón de comportamiento similar para cada grupo etario, observándose que en los varones el pliegue del tríceps y la circunferencia y el área adiposa del brazo se mantienen más o menos constantes en el intervalo de edad incluido en este estudio, mientras que en las mujeres el pliegue del tríceps y el área adiposa del brazo muestran dentro de cada grupo etario valores más elevados que en los varones y además se incrementan progresivamente con la edad, aumentando el área adiposa en mayor medida que el pliegue del tríceps ${ }^{34}$.

Esta diferencia de incremento entre el área adiposa del brazo y el pliegue del tríceps muestra que la circunferencia del brazo también aumenta con la edad en las mujeres, afectando al cálculo del área adiposa y también, y en mayor medida, al cálculo del área muscular, y podría explicar porqué las diferencias entre el $\% \mathrm{GC}_{\mathrm{BRAzO}}$ y el resto de métodos disminuyen con la edad. También justificaría la paradoja observada en estos estudios antropométricos de que en las mujeres el área muscular aumente con la edad, ya que, al aumentar el depósito de gra- 
sa a nivel del brazo y comportarse este como un cilindro, aumenta la superficie del brazo, es decir, su circunferencia, aumentando de forma paralela el valor del perímetro muscular y del área muscular del brazo, que no reflejarían en esta situación un incremento de masa muscular sino de masa grasa. Efecto que ya ha sido descrito en otros estudios ${ }^{22}$, en los que la circunferencia del brazo se ha mostrado más estrechamente relacionada con la masa grasa que con la masa libre de grasa del brazo y que deberá ser tenido en cuenta en el diseño de estudios epidemiológicos que incluyan estos parámetros.

Corroborando estos datos, estudios realizados mediante TAC muestran que el área muscular del brazo sobrestima en un 15$25 \%$ la masa muscular, sugiriéndose la introducción de un factor corrector para su cálcu$10^{35}$; aplicando este factor, el $\% \mathrm{GC}_{\text {BRAZO }}$ ofrece un valor mas elevado que hace más improbable su intercambiabilidad con el resto de técnicas.

Por otro lado, estudios realizados en cadáveres ${ }^{36}$ observan una buena correlación entre el grosor del pliegue del tríceps medido con el lipocalibrador y la profundidad de la incisión hasta la fascia muscular subyacente realizada en ese punto, no encontrando diferencias de compresibilidad entre varones y mujeres, por lo que este factor no estaría implicado en las diferencias observadas.

Entre las técnicas que alcanzan el grado de acuerdo bueno, la determinación de $\% \mathrm{GC}_{\text {TRÍCEPS }}$ requiere un menor número de pasos que $\% \mathrm{GC}_{\text {SIRI }}$ y posibilita disponer a nivel clínico de una herramienta más asequible para calcular este parámetro, dentro de las limitaciones inherentes a la medición de los pliegues cutáneos ${ }^{37}$. También se observa que la concordancia clínica entre $\% \mathrm{GC}_{\text {SIRI }} \mathrm{y}$ $\% \mathrm{GC}_{\text {TRí́cePs }}$ cuando se utilizan los coeficientes C y M específicos para cada grupo de edad es mayor que la reflejada en un estudio previo $^{17}$ utilizando los cuatro pliegues con los coeficientes $\mathrm{C}$ y M globales, al presentar una menor media de las diferencias y un menor intervalo de concordancia, por lo que para estimar el porcentaje de grasa corporal es preferible utilizar el pliegue del tríceps con los coeficientes específicos que medir los cuatro pliegues y utilizar los coeficientes globales.

Por último, los resultados obtenidos con el monitor Omron BF 300 permiten también considerar esta técnica como una alternativa válida frente a la utilización del pliegue del tríceps o de la suma de cuatro pliegues cutáneos. La impedanciometría es una técnica no invasiva ni molesta para el paciente, fácil de realizar, con buena fiabilidad ${ }^{19,20}$ y bajo coste, siendo previsible, como apunta el Consenso SEEDO' $2000^{12}$, que su utilización se incremente.

Como resumen, cuando se calcula a partir de las áreas del brazo el porcentaje de grasa corporal se produce una sobreestimación respecto al valor obtenido por impedanciometría o mediante la ecuación de Siri para cuatro pliegues o el pliegue del tríceps, influyendo en las diferencias observadas el número de parámetros antropométricos presentes en la ecuación, la gestión que de dichos parámetros se hace en cada una de las ecuaciones utilizadas y factores constitucionales modulados por la edad, que hacen además que las diferencias sean mayores en las mujeres que en los varones.

Los resultados obtenidos sugieren que en ambos sexos las áreas del brazo no reflejan adecuadamente la composición corporal, en los varones de forma independiente al IMC y en las mujeres en mayor medida en las no obesas que en las obesas, y que el porcentaje de grasa estimado a partir de ellas puede no ser un indicador válido de la adiposidad global, sobre todo en mujeres. Aunque este planteamiento se ve apoyado por el hecho de que el porcentaje de grasa obtenido con este método es el que correlaciona en menor medida con el IMC, este extremo debe ser confirmado frente a una técnica patrón. 
Por último, la menor asociación con el IMC y la circunferencia de la cintura, parámetros asociados al riesgo metabólico-cardiovascular ${ }^{38}$, también limita su utilidad tanto a nivel clínico como epidemiológico.

\section{AGRADECIMIENTOS}

Al Dr. Manuel de Oya Otero, catedrático del Departamento de Medicina de la Universidad Autónoma de Madrid y jefe de servicio de la Unidad de Lípidos de la Fundación Jiménez Díaz, por sus aportaciones y comentarios.

\section{BIBLIOGRAFÍA}

1. Venegas Moreno E, Soto Moreno A, Pereira Cunill JL, García Peris P, León Sanz M, Pita Mercé AM, García-Luna PP. Pacientes en riesgo de desnutrición en asistencia primaria. Estudio sociosanitario. Nutr Hosp 2001; 16: 1-6.

2. Esteban Pérez M, Fernández-Ballart J, Salas-Salvadó J. Estado nutricional de la población anciana en función del régimen de institucionalización. Nutr Hosp 2000; 15: 105-113.

3. Ferrero López MI, Botella Trelis JJ. Alimentación y nutrición en la enfermedad de Alzheimer. Nutr Hosp 2000; 15: 280-290.

4. Izquierdo Villaroya B, Celaya Pérez S, Amiguet García JA. Estado nutricional del paciente VIH. Descripción y evolución durante un año de un grupo de pacientes. Nutr Hosp 2000; 15: 302- 311.

5. Kumar NB, Cantor A, Allen K, Cox CE. Android obesity at diagnosis and breast carcinoma survival: Evaluation of the effects of anthropometric variables at diagnosis, including body composition and body fat distribution and weight gain during life span, and survival from breast carcinoma. Cancer 2000; 88: 2751-2757.

6. Van den Ham ECH, Coman JP, Christiaans MHL, Nieman FHM, Van Kreel BK, Heidendal GAK, Van Hooff JP. Body composition in renal transplant patients. J Am Soc Nephrol 1999; 10: 1067 1079.

7. de Miguel Díez J, Grau Carmona T, Izquierdo Alonso JL. Papel de la nutrición en la enfermedad pulmonar obstructiva crónica. Med Clin (Barc) 1998; 110: 307-316.

8. Brodie D, Moscrip V, Hutcheon R. Body Composition Measurement: A Review of Hydrodensitometry, Antrhopometry, and Impedance Methods. Nutrition 1998; 14: 296-310.

9. Ellis KJ. Human body composition: in vivo methods. Phys Rev 2000; 80: 649-680.

10. Gartner A, Maire B, Traissac P, Massamba JP, Kameli Y, Keraudren V, Delpeuch F. Sensitivity and specificity of the body mass index to assess low percent body fat in African women. Am J Human Biol 2000; 12: 25-31.

11. Kyle UG, Morabia A, Slosman DO, Mensi N, Unger P, Pichard C. Contribution of body composition to nutritional assessment at hospital admission in 995 patients: a controlled population study. Br J Nutr 2001; 86: 725-731.

12. Sociedad Española para el Estudio de la Obesidad (SEEDO). Consenso SEEDO’2000 para la evaluación del sobrepeso y la obesidad y el establecimiento de criterios de intervención terapéutica. Med Clin (Barc) 2000; 115: 587-597.

13. Allison DB, Zannolli R, Faith MS, Heo M, Pietrobelli A, VanItallie TB, Pi-Sunyer FX, Heymsfield SB. Weight loss increases and fat loss decreases all-cause mortality rate: results from two independent cohort studies. In J Obes Realt Metab Disord 1999; 23: 603-611.

14. Sociedad Española para el Estudio de la Obesidad (SEEDO). Consenso español 1995 para la evaluación de la obesidad y para la realización de estudios epidemiológicos. Med Clin (Barc) 1996; 107: 782-787.

15. Durnin JVGA, Womersley J. Body fat assessed from total body density and its estimation from skinfold thickness: measurements on 481 men and women aged from 16 to 72 years. Br J Nutr 1974; 32: 77-97.

16. Núñez C, Carvajal A, Turrero E, Moreiras O. Contribución al estudio de la composición corporal de un grupo de mujeres jóvenes mediante análisis de impedancia bioeléctrica. Nutr Hosp. 1994; 9: 262267.

17. Martín Moreno V, Gómez Gandoy JB, Antoranz González MJ. Medición de la grasa corporal mediante impedancia bioeléctrica, pliegues cutáneos y ecuaciones a partir de medidas antropométricas. Análisis comparativo. Rev Esp Salud Pública 2001; 75: 221-236.

Rev Esp Salud Pública 2003, Vol. 77, N. ${ }^{\circ} 3$ 
18. Alastrué A, Rull M, Camps I, Salvá JA. Nuevas normas y consejos en la valoración de los parámetros antropométricos en nuestra población: índice adiposo muscular, índices ponderales y tablas de percentiles de los datos antropométricos útiles en una valoración nutricional. Med Clin (Barc) 1988; 91: 223-236.

19. Martín Moreno V, Antoranz González MJ, Fernández Herranz S, Morales Barrios P, García González A. Factores clínicos y biológicos que influyen en la medición de la grasa corporal mediante bioimpedianciometría con el monitor Omron BF 300. Endocrinología y Nutrición 2001; 48: 259-265.

20. Martín Moreno V, Gómez Gandoy JB, Antoranz González MJ, Fernández Herranz S, Gómez de la Cámara A, de Oya Otero M. Validación del monitor de medición de la grasa corporal por impedancia bioeléctrica OMRON BF 300. Aten Primaria 2001; 28: 174-181.

21. Bland JM, Altman DG. Statistical methods for assessing agreement between two methods of clinical measurement. Lancet 1986; 1: 307-310.

22. Reid IR, Evans MC, Ames R. Relationships between upper-arm antrhopometry and soft-tissue composition in postmenopausal women. Am J Clin Nutr 1992; 56: 463-466.

23. Bracco D, Thiébaud D, Chiolero RL, Landry M, Burckhardt P, Schutz Y. Segmental body composition assessed by bioelectrical impedance analysis and DEXA in humans. J Appl Physiol 1996; 81: 2580-2587.

24. Biggs J, Cha K, Horch K. Electrical resistivity of the upper arm and leg yields good estimates of whole body fat. Psysiol Meas 2001; 22: 1-12.

25. Gibson AL, Heyward VH, Mermier CM. Predictive accuracy of Omron body logic analyzer in estimating relative body fat of adults. Int J Sport Nutr Exerc Metab 2000; 10: 216-227.

26. Esquius M, Schwartz S, López Hellín J, Andreu AL, García E. Parámetros antropométricos de referencia de la población anciana. Med Clin (Barc) 1993; 100: 692-698.

27. Burgert SL, Anderson CF. An evaluation of upper arm measurements used in nutritional assessment. Am J Clin Nutr 1979; 32: 2136-2142.

28. Deurenberg P, Yap M, van Staveren WA. Body mass index and percent body fat: a meta analysis among different ethnic groups. Int J Obes Relat Metab Disord 1998; 22: 1164-1171.
29. Deurenberg P, Andreoli A, Borg P, Kukkonen-Hrajula K, de Lorenzo A, van Marken WD et al. The validity of predicted body fat percentage from body mass index and from impedance in samples of five European populations. Eur J Clin Nutr 2001; 55: 973-979.

30. Morrison JA, Barton BA, Obarzanek E, Crawfordm PB, Guo SS, Schreiber GB. Racial differences in the sums of skinfolds and percentage of body fat estimated from impedance in black and white girls, 9 to 19 years of age: the National Heart, Lung, and Blood Institute Growth and Health Study. Obes Res 2001; 9: 297-305.

31. Ricart W, González-Huix F, Conde V y Grup per 1'Evaluació de la Composició Corporal de la Població de Catalunya. Girona. Valoración del estado de nutrición a través de la determinación de los parámetros antropométricos: nuevas tablas en la población laboral de Cataluña. Med Clin (Barc) 1993; 100: 681-691.

32. Casas YG, Schiller BC, de Souza CA, Seals DR. Total and regional composition across age in healthy Hispanic and white women of similar socioeconomic status. Am J Clin Nutr 2001; 73: 13-18.

33. Lean MEJ, Han TS, Deurenberg P. Predicting body composition by densitometry from simple anthropometric measurements. Am J Clin Nutr 1996; 63: 4-14.

34. Alastrué A, Sitges A, Jaurrieta E, Sitges A. Valoración antropométrica del estado de nutrición. Normas y criterios de desnutrición y obesidad. Med Clin (Barc) 1983; 80: 691-699.

35. Heymsfield SB, McManus C, Smith J, Stevens V, Nixon DW. Anthropometric measurements of muscle mass: revised equations for calculating bone-free arm muscle-area. Am J Clin Nutr 1982; 36 : 680-690.

36. Fernández Vieitez JA, García Suárez RM, Rodríguez Clemente CM. Compresibilidad del pliegue cutáneo y su relación con algunas variables antropométricas. Evidencias en cadáveres humanos. Rev Cubana Aliment Nutr 1999; 13: 18-23.

37. Ulijaszek SJ, Kerr DA. Anthropometric measurement error and the assessment of nutritional status. Br J Nutr 1999; 82: 165-177.

38. Expert Panel on the Identification, Evaluation and Treatment of Overweight in Adults. Clinical Guidelines on the Identificatión, Evaluation, and Treatment of Overweight and Obesity in Adults: Executive Summary. NHLBI Clinical Guidelines Executive Summary. Am J Clin Nutr 1998; 68: 899-917. 